\title{
In vivo self-assembled siRNA: a new modality for combination therapy of ulcerative colitis
}

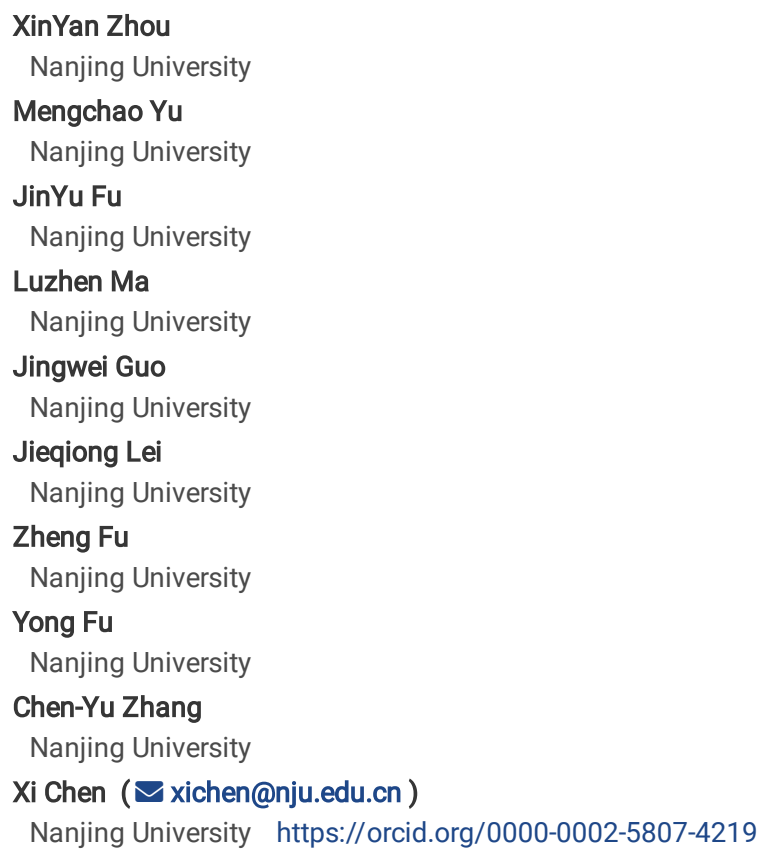

Keywords: combination therapy, siRNA, exosomes, UC

Posted Date: August 13th, 2021

DOI: https://doi.org/10.21203/rs.3.rs-765327/v1

License: @ (i) This work is licensed under a Creative Commons Attribution 4.0 International License. Read Full License

Version of Record: A version of this preprint was published at Nature Communications on September 28th, 2022. See the published version at https://doi.org/10.1038/s41467-022-33436-0. 


\section{Abstract}

Given the complex nature of ulcerative colitis (UC), combination therapy targeting multiple pathogenic genes and pathways of UC may be required. Unfortunately, current therapeutic strategies based on independent chemical compounds or monoclonal antibodies are not applicable for combination therapy of UC. Here, we developed a synthetic biology strategy that integrates the naturally existing exosome-circulating system with artificial genetic circuits for selfassembly and delivery of multiple siRNAs for the combination therapy of UC. Intravenous injection of genetic circuit (in the form of DNA plasmid) designed for inhibition of TNF-a, B7-1 and integrin a4 successfully reprogrammed the host liver to direct the self-assembly of TNF-a, B7-1 and integrin a4 siRNA into secretory exosomes. The multitargeted genetic circuit could rapidly relieved intestinal inflammation and exert a synergistic therapeutic effect against UC through suppressing the proinflammatory cascade in colonic macrophages, inhibiting the costimulatory signal to $\mathrm{T}$ cells and blocking $\mathrm{T}$ cell homing to sites of inflammation. More importantly, we designed an AAV-driven genetic circuit to induce substantial and lasting inhibition of TNF-a, B7-1 and integrin a4. Overall, this study established a feasible combination therapeutic strategy for UC, which is superior to the conventional biological therapies requiring.

\section{Introduction.}

Ulcerative colitis (UC) is a type of chronic and relapsing inflammatory bowel disease (IBD) that is characterized by uncontrolled intestinal inflammation and disruption of the colonic epithelial layer. ${ }^{1}$ Over the past decade, biological therapy with antibodies targeting to the excessive immune responses has been intensively investigated as a new therapeutic option for UC patients, especially in cases where the disease is refractory or intolerant to conventional agents. ${ }^{2}$ Based on the functions and mechanisms of the targets that are involved in the immune dysregulation of UC, current biological therapy can be classified into several subtypes. (1) Inhibition of proinflammatory cytokines or augmentation of the effect of anti-inflammatory cytokines. ${ }^{3}$ The most representative one is infliximab, a chimeric monoclonal antibody $(\mathrm{mAb})$ to human tumour necrosis factor-a (TNF-a), which has become the first-line treatment for moderate to severe UC. ${ }^{4}$ Infliximab promotes anti-inflammatory effects mainly by inhibiting TNF-a in colonic macrophages and suppressing a proinflammatory cascade, thereby relieving structural damage to the mucosa. ${ }^{5}$ (2) Blockade of T cell homing to sites of inflammation. Upon activation, $T$ cells express integrin a4 $\beta 7$ to recognize mucosal addressin cellular adhesion molecule-1 (MAdCAM-1), which is expressed at sites of inflamed vascular endothelium, thereby directing the migration of effector $\mathrm{T}$ cells to the intestinal lamina propria. ${ }^{6}$ Natalizumab, an antibody directed against integrin a4, can inhibit $\mathrm{T}$ cell migration through the endothelial vascular barrier, therefore showing clinical efficacy for the management of UC. ${ }^{7}$ (3) Blockade of T cell activation. Complete T cell activation requires two signals: the first is mediated by the interaction of the T cell receptors (CD4 or CD3) with the antigenic peptides presented by major histocompatibility complex (MHC) molecules on the surface of antigen presenting cells (APCs), and the second is the costimulatory signal, which is provided by the interaction between CD28 protein on the T cell surface and B7 protein (CTLA-4) on the membrane of APCs. ${ }^{8}$ Abatacept (a CLTA-4/Fc-fusion protein containing a high-affinity binding site for B7) inhibits the costimulatory signal to T cells, resulting in T cell anergy. ${ }^{9}$ Although biological therapy has yielded promising therapeutic outcomes in the preclinical and clinical treatment of UC, it still suffers from some inherent limitations of antibody drugs. The biggest problem is that antibody drugs have strong immunogenicity. A large portion of patients fail to respond to or gradually develop resistance to antibody therapy because of the generation of anti-antibodies. ${ }^{10}$ Simultaneously, the high treatment costs and serious side effects remain a major obstacle. Therefore, there is still a considerable unmet medical need to develop a new therapeutic approach for UC. ${ }^{11}$

Because UC is a multifactorial and multistep process, merely blocking a single inflammatory cytokine or immunological target may not be adequate and certainly not optimal to maintain clinical remission and achieve long-term efficacy. ${ }^{12,13}$ Recently, combination therapy has been proposed as a promising new treatment for a variety of diseases. Theoretically, a combination of two or more drugs given as a single formulation should be more effective than treatment with single or sequentially administered drugs. ${ }^{14}$ Unfortunately, it is still technically immature to use combination therapy in UC, especially for patients showing immunological heterogeneity and phenotypic variation. More importantly, traditional combination therapy based on the simultaneous administration of multiple drugs is costly and may involve complicated treatment regimens, undesired drug-drug interactions and an accumulative risk of side effects. ${ }^{13}$ Due to the high specificity, potency and flexibility of small interfering RNAs (siRNAs) to control multiple pathogenic genes and pathways at a single dosage, RNA interference (RNAi)-based therapeutics offer an attractive strategy to cotarget multiple pathways involved in the pathogenesis of UC. ${ }^{15}$ In principle, the design of a combinatory expression cassette that carries multiple siRNA-expressing units can cotranscribe these siRNAs in cells, thus providing a simple and efficient strategy for inhibiting multiple genes simultaneously. However, siRNAs are susceptible to degradation by ubiquitous RNases and, as they are anionic and hydrophilic, are unable to cross the cell membrane efficiently. ${ }^{16}$ Thus, the success of RNAi therapy relies heavily on siRNA carriers and delivery approaches. Currently, the development of an appropriate delivery system for siRNAs remains a major bottleneck of RNAi therapy. Recently, exosomes, nanosized membrane-bound extracellular vesicles self-produced by endogenous cells, have emerged as promising delivery carriers for siRNAs. ${ }^{17}$ However, it is still very difficult to load sufficient siRNA cargos into exosomes and harvest sufficient exosomes to reach the scale required by clinical practice. Recently, we developed a synthetic biology strategy that reprogrammes the host liver as a tissue chassis to direct the self-assembly of siRNAs into secretory exosomes and facilitate the in vivo delivery of siRNAs to desired tissues based on the intrinsic capability of the liver to express transgenes introduced by intravenous injection of genetic circuits (in the form of naked DNA plasmids). ${ }^{18}$ This state-of-the-art technology borrows the body's workshop and reconceptualizes the assembly and delivery strategies of siRNA-encapsulating exosomes, thereby avoiding the complicated procedures, high cost and labour intensity associated with current techniques for exosome engineering and delivery. Since self-assembled siRNAs hold the potential to simultaneously and synergistically target multiple genes, this technology may provide a promising tool to cotarget multiple genes and pathways in the aetiology of UC, thereby addressing the bottleneck problem in the treatment of UC. In this study, we designed a combinatory multitargeted genetic circuit and evaluated its therapeutic value in acute and chronic UC models.

\section{Materials And Methods.}

\section{Animals}


All the animal experiments were approved by Animal Ethical and Welfare Committee of NJU. Six-week-old male BALB/c mice were purchased from the Model Animal Research Center of Nanjing University and maintained under specific pathogen-free conditions at Nanjing University.

\section{Design and construction of the genetic circuits}

The CMV-siR ${ }^{\text {TNF-a }}$ circuit was generated by inserting a TNF-a siRNA sequence (5'-CCATTTGGGAACTTCTCATCC-3') into a 166-bp pre-miR-155 backbone with structurally conserved nucleotide changes to maintain pairing (GGATCCTGGAGGCTTGCTGAAGGCTGTATGCTGAATTCGCCATTTGGGAACTTCTCATCCGTTTTGGCCACTGACTGACGGATGAGATTCCCAAATGGCAACCGGTCAG the CMV-siR ${ }^{T+B+1}$ circuit designed to co-express TNF-a siRNA, B7-1 siRNA and integrin a4 siRNA, two tandem pre-miR-155 backbones carrying the B7-1 siRNA sequence ( $5^{\prime}$ - AAGAGAAACTAGTAAGAGTCT-3') and the integrin a4 siRNA sequence (5'- ATCACATGATGCCCAAGGTGG -3') were cloned downstream of the TNF-a siRNA element. A circuit designed to express a scrambled RNA was used as the negative control. The plasmids used to express genetic circuits were synthesized and constructed by Realgene Biotech Company (Nanjing, China). Maps and scaffolds of the plasmids are shown in Supplementary Fig. 17. The plasmids were transformed into E. coli DH5a competent cells (Tsingke, TSC01, Beijing, China), cultured with LB medium (with $50 \mu \mathrm{g} / \mathrm{mL}$ spectinomycin) for 14 hours in a $37^{\circ} \mathrm{C}$ shaking incubator and extracted and purified with an EndoFree Maxi Plasmid Kit V2 (Tiangen, DP120, Beijing, China) according to the manufacturer's instructions. The purified plasmids were sequenced to ensure that the sequences of the inserted genetic circuits were correct.

Sequences of the siRNAs designed for silencing of TNF-a, IL-17A, INF-y, IL-6, integrin a4, ICAM-1, CD3 and B7-1 are shown in Supplementary Table. 1. Sequences of the siRNA expression cassettes are shown in Supplementary Table. 2.

For construction of the AAV-driven genetic circuits, the complete sequences of the CMV-siR ${ }^{T N F-a}$ and $C M V$-siR ${ }^{T+B+1}$ backbones were inserted into the $A A V-9$ vector by HANBIO (Wuhan, China).

\section{Extraction and culture of primary cells}

Peritoneal macrophages were collected from the DSS-induced UC mice by lavage from the peritoneal cavity using $5 \mathrm{~mL}$ ice-cold PBS. ${ }^{19}$ ANA-1 cells and peritoneal macrophages were cultured at $37^{\circ} \mathrm{C}$ under $5 \% \mathrm{CO}_{2}$ in RPMI -1640 supplemented with $10 \%$ foetal bovine serum (FBS, Gibco, MA, USA).

Mononuclear cells were isolated from the peripheral blood and spleen by using mouse peripheral blood mononuclear cells separation kit (TBD science, LDS1090, Tianjin, China) and mouse splenic mononuclear extraction kit (TBD science, LDS1090PK), respectively. Lamina propria mononuclear cells were isolated using a method as described previously. ${ }^{20}$ Briefly, the colon was removed from the sacrificed mice, cut into $0.5 \mathrm{~cm}$ pieces and washed thoroughly with cold PBS to remove all debris and blood. After incubating with $2 \mathrm{mM}$ dithiothreitol and $1 \mathrm{mM}$ EDTA in PBS at $37^{\circ} \mathrm{C}$ for $2 \times 20$ minutes under gentle shaking to remove intestinal epithelial cells, the tissues were digested in $10 \mathrm{~mL} 2 \%$ FBS-RPMI-Collagenase $\mathrm{A}\left(1 \mathrm{mg} / \mathrm{mL}\right.$, Roche, Mannheim, Germany) at $37^{\circ} \mathrm{C}$ for 30 minutes. Lamina propria cells were then collected and further purified via density gradient centrifugation with $40 \%$ and $70 \%$ Percoll-RPMI solution. Lamina propria mononuclear cells were collected from the interphase.

Monocytes were isolated from the peripheral blood mononuclear cells using the peripheral blood monocyte extraction kit (TBD science, TBD2011M). Splenic macrophages and colonic macrophages were isolated by using anti-F4/80 MicroBeads UltraPure (Miltenyi Biotec, 130-110-443, Bergisch gladbach, Germany) from spleen mononuclear cells and lamina propria mononuclear cell.

Peripheral blood lymphocytes and spleen lymphocytes were enriched using mouse peripheral blood lymphocyte separation kit (TBD science, LTS1092) and mouse splenic lymphocyte extraction kit (TBD science, LTS1092PK). CD4 ${ }^{+}$T cells were obtained from peripheral blood lymphocytes, spleen lymphocytes andlamina propria mononuclear cells with $\mathrm{CD}^{+} \mathrm{T}$ cell Isolation kit (Miltenyi Biotec, 130-104-454, Bergisch gladbach, Germany).

All primary cell extraction steps were performed under aseptic conditions, and the viability of the primary cells was determined by trypan blue staining and cell count.

\section{Statistical analysis}

Details on the statistics used can be found in the figure legends. All statistical analyses were performed using commercially available software (GraphPad Prism 9). Data were first checked for a normal distribution, differences among groups were compared by one-way ANOVA, and multiple comparisons were conducted by Dunnett's test. $\mathrm{N}$ represents the number of samples used in the experiments. Data are means with error bars showing the SEM. Significance was assumed at * $p<0.05 ; * \star p<0.01 ; * \star * p<0.005$.

Additional information is available in Supplementary Materials and Methods.

\section{Results.}

Construction and characterization of the genetic circuits targeting TNF- $a$ 
We evaluated the therapeutic effects of the genetic circuit designed to specifically target TNF-a in colonic macrophages for the treatment of UC. We constructed a genetic circuit consisting of two functional modules: the promoter module drives the transcription of siRNA and directs the package of siRNA into exosomes, while the siRNA expression cassette module maximizes the expression of the siRNA guide strand and minimizes the expression of undesired passenger strand. Based on our previous study, the cytomegalovirus (CMV) promoter was selected as the promoter module, and the pre-miR-155 backbone was selected as the optimal siRNA expression cassette to produce siRNA. ${ }^{18}$ By inserting the TNF-a siRNA sequence into the $5^{\prime}$ arm of the pre-miR-155 hairpin, a CMV-directed genetic circuit (in the form of a DNA plasmid) carrying a TNF-a siRNA expression cassette was constructed (hereafter, the CMV-siR ${ }^{\text {TNF-a }}$ circuit) (Fig. 1a).

Next, we tested whether the CMV-siR ${ }^{\text {TNF-a }}$ circuit has the ability to synthesize functional TNF-a siRNA in vitro. ANA-1 cells were transfected with three CMV$\mathrm{siR}^{\mathrm{TNF}-\mathrm{a}}$ circuits designed to target different sites of the coding sequence (CDS) of TNF-a and then treated with LPS to stimulate an inflammatory response. A CMV-directed genetic circuit encoding a scrambled RNA (CMV-scrR circuit) was transfected as a negative control. ANA-1 cells exhibited a remarkable increase in TNF-a mRNA expression with LPS stimulation; however, this effect was considerably reduced in ANA-1 cells transfected with the three CMV-siR ${ }^{\text {TNF-a }}$ circuits (Supplementary Fig. 1a). Likewise, while LPS dramatically stimulated the secretion of TNF-a into the cell culture medium, the three CMV-siR ${ }^{T N F-a}$ circuits significantly suppressed the amounts of secreted TNF-a (Supplementary Fig. 1b). The circuit with the greatest interference efficiency, CMV-siR ${ }^{\text {TNF-a }}-1$, was selected for further evaluation.

\section{Evaluation of the self-assembly of TNF-a siRNA-encapsulating exosomes in an ex vivo model}

Subsequently, we established an ex vivo model to examine the self-assembly and secretion of TNF-a siRNA-encapsulating exosomes. An acute UC model was induced in male BALB/c mice by replacing their drinking water with a $2.5 \%$ DSS solution for 7 days; the CMV-scrR or CMV-siR ${ }^{\text {TNF-a }}$ circuit was intravenously injected into DSS mice 7 times, and then, the exosomes were purified from mouse plasma and incubated with in vitro cultured macrophages (Fig. 2a). First, we confirmed the proper enrichment of exosomes from mouse plasma by nanoparticle tracking analysis (NTA) and transmission electron microscopy

(TEM) (Supplementary Fig. 2). Second, we investigated whether the CMV-siR ${ }^{\text {TNF-a }}$ circuit could trigger the efficient packaging of TNF-a siRNA into exosomes. A

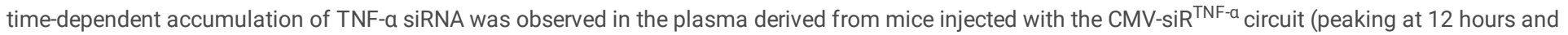
decreasing to a background level at 48 hours) (Fig. 2b). Interestingly, most TNF-a siRNA was detected in the plasma exosomes rather than exosome-free plasma of CMV-siR ${ }^{\text {TNF-a }}$-injected mice (Fig. 2c). These results suggested that TNF-a siRNA was released into the blood circulation in an exosome-dependent manner. Third, we evaluated whether the formation of TNF-a siRNA-encapsulating plasma exosomes could reduce TNF-a expression in macrophages. When macrophages were incubated with plasma exosomes derived from mice injected with the CMV-siR ${ }^{\text {TNF-a }}$ circuit and then stimulated with LPS, a significant decrease in TNF-a mRNA expression was observed (Fig. 2d), which was accompanied by a reduced amount of TNF-a secreted into the cell culture medium (Fig. 2e).

\section{Tracking of the delivery of self-assembled TNF-a siRNA to immune cells in inflamed mucosa}

We investigated whether exosome-enclosed TNF-a siRNA was successfully delivered to mononuclear phagocytes, particularly to colonic macrophages, via the exosome-circulating system. The CMV-siR ${ }^{T N F-a}$ circuit was intravenously injected into DSS-induced acute UC mice, and then the biodistribution of TNF-a siRNA in various tissues was determined. Time-dependent accumulation and clearance of TNF-a siRNA was observed in the livers of

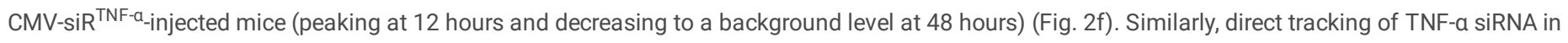
mouse liver by fluorescence in situ hybridization (FISH) also revealed a time-dependent change in TNF-a siRNA levels in the liver (Fig. $2 \mathrm{~g}$ and Supplementary Fig. 3a). These results are consistent with the findings of previous studies and support the idea that the liver can express transgenes introduced by intravenously injected genetic circuits (naked DNA plasmids). ${ }^{21}$ In addition to the liver, a time-dependent accumulation of TNF-a siRNA was also observed in the colon, spleen and kidney of CMV-siR ${ }^{\text {TNF-a }}$-injected mice (peaking at 12-24 hours and decreasing significantly after 48 hours) (Fig. $2 \mathrm{~h}$ ). Likewise, when the distribution of TNF-a siRNA to the colon was examined by FISH, hybridization signals of TNF-a siRNA were detected, with a similar time trend, in the colon sections of CMV-siR ${ }^{\text {TNF-a }}$-injected mice (Fig. $2 \mathrm{i}$ and Supplementary Fig. 3b). To further quantify the cellular uptake of TNF-a siRNA, immune cells

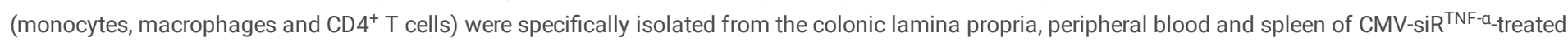
mice. A time-dependent increase in TNF-a siRNA levels was detected in these isolated immune cells (Fig. 2j and 2k). Thus, TNF-a siRNA-encapsulating exosomes possessed the capacity to access immune cells in inflamed mucosa, blood and spleen, resulting in efficient delivery of TNF-a siRNA to the sites of desire.

\section{In vivo therapeutic efficacy of self-assembled TNF-a siRNA in an acute UC model}

Next, we evaluated the therapeutic effects of self-assembled TNF-a siRNA on mice suffering from acute UC. An acute UC model was induced in male BALB/c mice by replacing their drinking water with a $2.5 \%$ DSS solution for 7 days. Starting on day 3, DSS mice were intravenously injected with CMV-scrR or three dosages of the CMV-siR ${ }^{\text {TNF-a }}$ circuit or infliximab once a day for seven times until the final analysis on day 10 (Fig. 3a). CMV-scrR circuit-treated DSS mice experienced apparent body weight loss compared with normal control mice; however, treatment with a high dose of the CMV-siR ${ }^{\mathrm{TNF}-\mathrm{a}}$ circuit significantly 
alleviated body weight loss in DSS mice (Fig. 3b). The disease activity index (DAl), a composite score reflecting the severity of the disease characteristics, was dramatically elevated in CMV-scrR circuit-treated DSS mice, but this score was significantly alleviated by treatment with a high dose of the CMV-siR ${ }^{\text {TNF-a }}$ circuit (Fig. 3c). Furthermore, CMV-scrR circuit-treated DSS mice had distinctly shorter colon lengths than normal mice, but treatment with a high dose of the CMV-siR ${ }^{T N F-a}$ circuit caused a significant recovery of colon length (Fig. $3 \mathrm{~d}$ and Supplementary Fig. 4a). In terms of the expression levels of TNF- $a$, while a remarkable increase in colonic TNF-a mRNA and protein levels was observed in the DSS mice treated with the CMV-scrR circuit, a dosedependent reduction in TNF-a mRNA and protein levels was observed in the colon of DSS mice injected with the CMV-siR ${ }^{\text {TNF-a }}$ circuit (Fig. $3 e$ and 3f). Immunofluorescence staining of TNF-a also confirmed a dose-dependent decline in TNF-a protein levels in the colonic lamina propria derived from CMV-

siR $^{\text {TNF-a }}$ circuit-treated mice (Fig. 3g and Supplementary Fig. 4b). Accordingly, while the production of proinflammatory cytokines (IL-6, IL-12p70, IL-17A and IL23) that are mechanistically associated with TNF-a was evoked in the colon of CMV-scrR circuit-treated DSS mice, all of these cytokines were markedly reduced by a high dose of the CMV-siR ${ }^{\text {TNF-a }}$ circuit (Supplementary Fig. 4c). Likewise, myeloperoxidase (MPO) activity, a direct indicator of the infiltration of neutrophils into colonic mucosa, was alleviated by a high dose of the CMV-siR ${ }^{\text {TNF-a }}$ circuit (Supplementary Fig. 4d). Finally, many cardinal histological signs of UC, including mucosal damage, ulceration, neutrophil infiltration, crypt abscesses and muscular layer thickness, were clearly manifested in the colonic

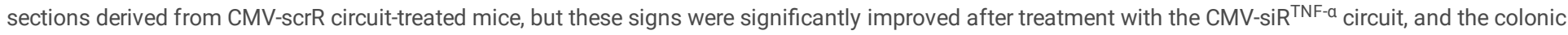
sections derived from CMV-siR ${ }^{\text {TNF-a }}$ circuit-treated mice had relatively intact epithelia, well-defined crypt structures and relatively low levels of neutrophil infiltration (Fig. 3h and Supplementary Fig. 4e). Accordingly, the colonic histological score was higher in CMV-scrR circuit-treated mice, but this score was dose-dependently reduced by treatment with the CMV-siR ${ }^{T N F-a}$ circuit (Supplementary Fig. 4f). Overall, these results demonstrated that the self-assembled TNF-a siRNA induced by the CMV-siR ${ }^{\text {TNF- }}$ circuit could alleviate the characteristics of DSS-induced inflammation and achieve mucosal healing. Conversely,

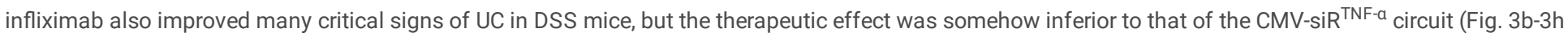
and Supplementary Fig. 4). Similarly, in an acute colitis model established by trinitrobenzene sulfonic acid (TNBS), the characteristic symptoms of colitis were significantly alleviated by treatment with the CMV-siR ${ }^{\mathrm{TNF}-\mathrm{a}}$ circuit, to a similar, if not better, extent as treatment with infliximab (Supplementary Fig. 5).

\section{In vivo therapeutic efficacy of self-assembled TNF-a siRNA in a chronic UC model}

Because UC is a chronic condition characterized by recurrent episodes of intestinal inflammation, we established a DSS-induced chronic UC

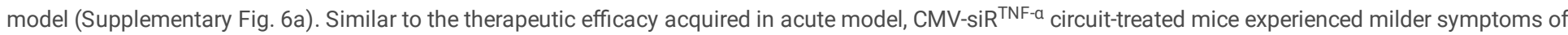
UC, including lower body weight loss, a decreased DAI score, a longer colon length, reduced colonic proinflammatory cytokines, reduced MPO activity and an improved histological appearance of colonic sections, compared with the control mice treated with the CMV-scrR circuit (Supplementary Fig. $6 \mathrm{~b}$-6g). At the molecular level, treatment with the CMV-siR ${ }^{\mathrm{TNF}-\mathrm{a}}$ circuit significantly reduced the expression levels of TNF-a mRNA and protein in the colon of the chronic UC model (Supplementary Fig. 6j-6l). The CMV-siR ${ }^{T N F-a}$ circuit performed slightly better than infliximab in ameliorating the clinical and histopathological severity of UC in the chronic model (Supplementary Fig. 6h-6i). Furthermore, the potential side effects and tissue toxicity of the genetic circuits were evaluated. Repeated injection of the CMV-siR ${ }^{\mathrm{TNF}-\mathrm{a}}$ circuit posed negligible hepatic toxicity and renal toxicity and caused no overt tissue damage in the chronic UC model (Supplementary Fig. 7a). Likewise, representative serum biochemical indexes, including alanine aminotransferase (ALT), aspartate aminotransferase (AST), total bilirubin (TBIL), blood urea nitrogen (BUN), alkaline phosphatase (ALP) and creatinine (CREA), were unchanged after treatment with the CMV-siR ${ }^{T N F-a}$ circuit (Supplementary Fig. 7b). In addition, the anti-TNF-a efficacy of the CMV-siR ${ }^{\text {TNF-a }}$ circuit was validated in a TNBS-induced chronic colitis model (Supplementary Fig. 8). Overall, these results demonstrate the therapeutic potential of the self-assembled TNF-a siRNA to inhibit the progression of intestinal inflammation and to promote the recovery of colon tissue from UC.

\section{Design of a multitargeted genetic circuit to simultaneously assemble multiple siRNAs for combination therapy of UC}

Subsequently, we designed a combinatory multitargeted genetic circuit to simultaneously block multiple causal genes and pathways relevant to the onset and persistence of UC. Based on literature mining, ${ }^{22-26}$ we first set up a group of candidate target genes, including proinflammatory cytokines (IL-17A, INF-y and IL6 ), ${ }^{22-25}$ adhesion molecules involved in T cell trafficking to the gut (integrin a4 and ICAM-1) ${ }^{26,27}$ and molecules essential for T cell activation (CD3 and B7$1)^{8,9}$. Second, we constructed a library of genetic circuits among which each genetic circuit carried only one siRNA expression cassette directed against one of the candidate target genes (Supplementary Table. 2). After assessing the therapeutic efficacy of these genetic circuits individually, genetic circuits targeting B7-1, integrin a4 and ICAM-1 were shown to be more effective than circuits targeting IL-17A, INF- $y$, IL- 6 and CD3 in ameliorating the manifestations of DSSinduced UC, as assessed by the DAI score and colon length (Supplementary Fig. 9a-9c). Moreover, genetic circuits targeting B7-1 and integrin a4 were more efficient than circuits targeting IL-17A, INF- $y$, IL-6, CD3 and ICAM-1 in knocking down their target genes (Supplementary Fig. 9d-9j). To view the effects as a whole, genetic circuits targeting B7-1 and integrin a4 were retained. Third, we constructed a multitargeted genetic circuit carrying three siRNA expression cassettes, which were organized into a head-to-tail tandem array under the control of a CMV promoter to simultaneously silence TNF-a, B7-1 and integrin a4 (hereafter, the CMV-siR ${ }^{T+B+1}$ circuit) (Fig. 1b). As a basis of comparison, the CMV-siR ${ }^{T N F-a}, C M V-s i R^{B 7-1}$ and CMV-siR ${ }^{\text {Integrin a4 }}$ circuits carrying a single siRNA expression cassette against their corresponding target genes were included as controls.

To validate that intravenous injection of the $\mathrm{CMV}$-siR ${ }^{T+B+1}$ circuit indeed induced the coassembly of three siRNAs into plasma exosomes and facilitated the delivery of three siRNAs to target cells, we assessed the in vivo distribution of the three siRNAs in a DSS-induced UC model. Regardless of injection with individual CMV-siR ${ }^{T N F-a}$ circuit or multitargeted CMV-siR ${ }^{T+B+1}$ circuit, a similar amount of TNF-a siRNA was detected in the plasma and colon of DSS mice 
(Supplementary Fig. 10a-10b). Direct tracking of TNF-a siRNA by FISH also revealed the clear presence of TNF-a siRNA in the colonic macrophages derived from the mice injected with the CMV-siR ${ }^{T N F-a}$ or CMV-siR ${ }^{T+B+1}$ circuit (Supplementary Fig. 10c). Simultaneously, a similar amount of B7-1 siRNA accumulated

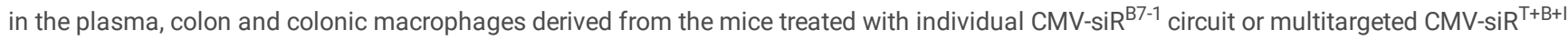

circuit (Supplementary Fig. 10d-10f). Moreover, compared with the DSS mice treated with the CMV-scrR circuit, extensive accumulation of integrin a4 siRNA was detected in the plasma, colon and colonic $\mathrm{CD}^{+} \mathrm{T}$ cells of DSS mice treated with the CMV-siR ${ }^{\text {Integrin a4 }}$ or CMV-siR ${ }^{\mathrm{T}+\mathrm{B}+1}$ circuit $(\mathrm{Supplementary} \mathrm{Fig.} 10 \mathrm{~g}$ $10 \mathrm{i})$.

Next, the therapeutic effects of the multitargeted CMV-siR ${ }^{T+B+1}$ circuit were evaluated in a DSS-induced chronic UC model to investigate whether a synergistic advantage was achieved (Fig. 4a). While both multi- and single-targeted genetic circuits significantly alleviated body weight loss, promoted the recovery of colon length, reduced the DAl score and improved the histological appearance in the DSS-induced UC model, the CMV-siR ${ }^{T+B+1}$-treated group showed the least body weight loss, best colon length recovery, lowest DAI score and inflammatory cytokine levels, and minimal histological signs of UC among the three treatment groups (Fig. 4b-4e and Supplementary Fig. 11). In terms of body weight, colon length and inflammatory cytokine levels, treatment with the CMV$\mathrm{SiR}^{\mathrm{T}+\mathrm{B}+1}$ circuit even alleviated these pathophysiological parameters to a similar level in normal control mice (Fig. 4b-4e and Supplementary Fig. 11). In particular, colon tissues from the CMV-siR ${ }^{T+B+1}$-treated group exhibited almost the same tissue morphology as that observed in the normal control group, especially with respect to the integration of the colonic epithelial layer and the infiltration of inflammatory cells (Fig. 4e and Supplementary Fig. 11c). Moreover, hepatic and renal toxicity and abnormal alterations in serum biochemical indexes were not observed after injection with the multitargeted CMV$\mathrm{siR}^{\mathrm{T}+\mathrm{B}+1}$ circuit (Supplementary Fig. 7). Overall, combination therapy with the multitargeted CMV-siR ${ }^{T+B+1}$ circuit yielded the highest therapeutic efficacy among all the tested groups, indicating that the multitargeted genetic circuit could exert a synergistic therapeutic effect against DSS-induced UC.

At the molecular level, the knockdown efficiency of the corresponding target genes was evaluated. First, a remarkable reduction in TNF-a mRNA and protein levels was observed in the colon of mice injected with the CMV-siR ${ }^{T N F-a}$ or CMV-siR ${ }^{T+B+1}$ circuit (Fig. 4f-4g). To further characterize the colonic macrophages that internalized TNF-a siRNA, primary macrophages were specifically isolated from the colonic lamina propria and cultured in conditioned medium for 12 hours. Immunofluorescence staining of TNF-a in primary macrophages revealed that TNF-a protein levels were significantly decreased by treatment with CMV$\mathrm{siR}^{\mathrm{TNF}-\mathrm{a}}$ or $\mathrm{CMV}$-siR ${ }^{\mathrm{T}+\mathrm{B}+1}$ circuit (Supplementary Fig. 12a). Accordingly, the number of colon-infiltrating macrophages, as measured by flow cytometric analysis

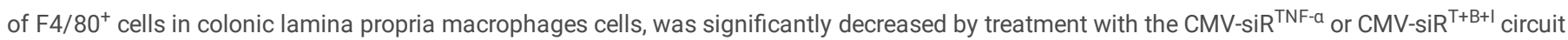
(Supplementary Fig. 12b-12c). Second, the levels of B7-1 mRNA were significantly reduced in the colon of mice injected with the CMV-siR ${ }^{B 7-1}$ or CMV-siR ${ }^{T+B+1}$ circuit (Fig. 4h). Flow cytometry also revealed that the colonic lamina propria mononuclear cells, peripheral blood mononuclear cells and splenic mononuclear cells isolated from DSS mice treated with the CMV-siR ${ }^{B 7-1}$ or CMV-siR ${ }^{T+B+1}$ circuit exhibited reduced amounts of B7-1 protein (Fig. 4i-4j and Supplementary Fig. 13a-13d). Immunofluorescence staining of B7-1 in primary cultured macrophages of DSS mice also confirmed an apparent reduction of B7-1 protein after treatment with the $\mathrm{CMV}$-siR ${ }^{\mathrm{B} 7-1}$ or $\mathrm{CMV}$-siR ${ }^{\mathrm{T}+\mathrm{B}+1}$ circuit (Supplementary Fig. 13e). Furthermore, double staining of B7-1 and F4/80 in colon sections of DSS mice revealed that the number of double-positive $\mathrm{B} 7-1^{+} \mathrm{F} 4 / 80^{+}$cells increased remarkably in $\mathrm{CMV}$-scrR circuit-treated DSS mice compared with normal mice, but this increase was abrogated by treatment with the CMV-siR ${ }^{B 7-1}$ or $C M V-s i R^{T+B+1}$ circuit, especially the latter (Fig. 4k and Supplementary Fig. $13 f$ ). These results suggest that B7-1-positive macrophages are increased at sites of intestinal inflammation, whereas intravenous injection of genetic circuits targeting B7-1 causes a synergistic decline in B7-1 protein and colon-infiltrating macrophages in inflamed mucosa. Since the B7-1 molecule on APCs provides costimulatory signals for $\mathrm{T}$ cell activation, we evaluated $\mathrm{T}$ cell activation using $\mathrm{CD} 25$ as a marker. ${ }^{28}$ Flow cytometry revealed that after $\mathrm{CMV}$-siR ${ }^{\mathrm{B} 7-1}$ or CMV-siR ${ }^{T+B+1}$ circuit treatment, the positive rate of $\mathrm{CD}_{2} 5^{+} \mathrm{T}$ cells was significantly reduced (Supplementary Fig. 13g-13h). Third, a significant

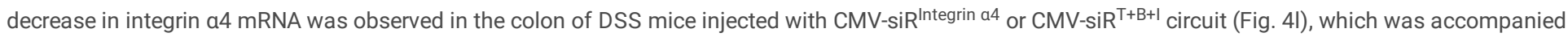
by a significant decline in integrin a4 protein levels in the membrane surface of lymphocytes derived from the colonic lamina propria, peripheral blood and spleen of these mice (Fig. $4 \mathrm{~m}-4 \mathrm{n}$ and Supplementary Fig. 14a-14d). In the immunofluorescence staining assay, double-positive signals of integrin a $4^{+}$CD $4^{+}$ cells were readily detected in the colon sections of CMV-scrR circuit-treated DSS mice; however, the double-positive signals were remarkably diminished by treatment with the CMV-siR Integrin a4 or CMV-siR ${ }^{T+B+1}$ circuit (Fig. 40 and Supplementary Fig. 14e). To investigate the interaction of integrin a4 $\beta 7$ with their ligands, a solid-phase adhesion assay was performed with plates coated with E-cadherin. ${ }^{26}$ The amounts of adherent CD $4^{+} \mathrm{T}$ cells isolated from the peripheral blood of CMV-siR ${ }^{\text {Integrin a4 }}$ circuit- or CMV-siR ${ }^{T+B+1}$ circuit-treated mice were significantly lower than those from CMV-scrR circuit-treated mice (Supplementary Fig. 14f-14g), indicating that homing of lymphocytes to the inflamed gut mucosa was abolished by treatment with the $\mathrm{CMV}^{\mathrm{si}} \mathrm{R}^{\mathrm{Integrin}} \mathrm{a} 4$ and $\mathrm{CMV}$-siR ${ }^{\mathrm{T}+\mathrm{B}+\mathrm{I}}$ circuit.

\section{Development of an AAV-based strategy for long-term self-assembly and delivery of exosome-enclosed siRNAs for the treatment of UC}

Next, we focused on optimizing the delivery vehicles of genetic circuits to achieve a long-term therapeutic effect. Since adeno-associated virus (AAV) is clinically safe and capable of establishing long-term transgene expression, we sought to investigate whether AAV-based liver delivery and expression of a genetic circuit enabled long-term self-assembly of siRNAs in the liver and induced constant target gene silencing in vivo. ${ }^{29}$ We inserted the whole sequence of the CMV-siR ${ }^{\text {TNF-a }}$ circuit into an AAV serotype 9 (AAV-9) vector (AAV-CMV-siR ${ }^{\text {TNF-a }}$ ) and evaluated the therapeutic effects in a chronic UC model (Supplementary Fig. 15a). Since the AAV-9 vector could coexpress TNF-a siRNA and a luciferase reporter, evaluation of luciferase activity might reflect TNF-a siRNA accumulation in vivo. AAV-mediated luciferase expression was dose-dependently increased from week 1, reached a peak at week 3 and decreased to 
the background level at week 10 (Supplementary Fig. 15b-15c). Compared with treatment with AAV-CMV-scrR, treatment with a high dose of AAV-CMV-siR ${ }^{\text {TNF-a }}$ caused a significant recovery of body weight and colon length, a significant decline in the DAI score and proinflammatory cytokine levels and an apparent alleviation of the histological appearance in DSS mice (Supplementary Fig. 15d-15i). At the molecular level, treatment with a high dose of AAV-CMV-siR ${ }^{\text {TNF-a }}$ resulted in a significant accumulation of TNF-a siRNA in plasma, and consequently, an apparent reduction in TNF-a mRNA and protein levels was observed in colon tissue (Supplementary Fig. 15j-15I). These results revealed that AAV-mediated liver expression of a genetic circuit provided a substantial and lasting therapeutic effect following a single administration.

Finally, the multitargeted CMV-siR ${ }^{T+B+1}$ circuit was inserted into the AAV-9 vector (AAV-CMV-siR ${ }^{T+B+1}$ ) to induce long-term combination therapy in a chronic UC model (Fig. 5a). AAV-CMV-siR ${ }^{T+B+1}$-treated mice recovered body weight quite fast after each DSS administration, especially those in the high-dose group (Fig.

$5 b)$. Likewise, $A A V-C M V$-siR ${ }^{T+B+1}$-treated mice experienced a significant recovery in colon length, had a dramatic decrease in the DAI score and proinflammatory cytokine levels and displayed a striking improvement in histological signs compared with mice treated with AAV-CMV-scrR (Fig. 5c-5e and Supplementary Fig. 16a-16d). Remarkably, while colon tissues from the AAV-CMV-scrR-treated mice exhibited epithelial disruption, goblet cell depletion and significant infiltration of inflammatory cells into the mucosa, colons from the AAV-CMV-siR ${ }^{T+B+1}$-treated mice showed a relatively normal histology, with no clear signs of inflammation or disruption of tissue morphology (Fig. 5e and Supplementary Fig. 16c). At the molecular level, treatment with AAV-CMV-siR ${ }^{T+B+1}$ resulted in a dose-dependent increase in TNF-a siRNA, B7-1 siRNA and integrin a4 siRNA in the liver, plasma and colon of DSS mice (Fig. 5f-5h and Supplementary Fig. 16e-16j), which was accompanied by a dose-dependent reduction in TNF-a, B7-1 and integrin a4 in colon tissues (Fig. 5i-5k).

\section{Discussion.}

Combination therapy is emerging as an attractive therapeutic option for UC. ${ }^{30}$ The basic concept behind this strategy is quite straightforward: simultaneous engagement of two or more different targets by a single drug formulation may have a synergistic therapeutic effect. ${ }^{31}$ However, monoclonal antibodies are usually used alone rather than in combination with other monoclonal antibodies, considering both the possibility of an increased risk of immunogenicity and side effects. Conversely, because the design of siRNAs is quite simple and flexible, RNAi-based combination therapy conducted by the codelivery of multiple siRNAs enables the simultaneous blocking of two or more different target genes or pathways in a single treatment. ${ }^{18}$ In this study, we developed an upgraded and innovative RNAi strategy through the design of a multitargeted CMV-siR ${ }^{\mathrm{T}+\mathrm{B}+1}$ circuit carrying a siRNA expression cassette organized as a head-to-tail tandem array under the control of a single CMV promoter. This multitargeted circuit reprogrammes the host liver to direct the self-assembly of TNF-a siRNA, B7-1 siRNA and integrin a4 siRNA into secretory exosomes and facilitate the in vivo delivery of these siRNAs to colonic macrophages and CD ${ }^{+} \mathrm{T}_{\text {cells. The }}$ improvements of the multiple-target strategy are reflected by two findings. First, in mouse models of UC, the multitargeted circuit exerted strong inhibitory activities on each of the corresponding target genes and exhibited a synergistic and cooperative therapeutic effect compared with the single-targeted circuit. Second, the multitargeted $\mathrm{CMV}$-siR ${ }^{\mathrm{T}+\mathrm{B}+1}$ circuit could simply decrease the dosages of plasmid and AAV carrying the genetic circuit when compared with the coinjection of three kinds of single-targeted circuits, thereby contributing to a reduction in the cytotoxicity, immunogenicity and side effects caused by the plasmid and AAV themselves. Overall, the multitargeted genetic circuit offers an easy-to-use, effective and safe combination therapeutic RNAi strategy, which is superior to conventional biological therapies where two or more independent compounds or antibodies are needed.

Considering the short in vivo half-life of the genetic circuits in the plasmid backbone, repeated injection of genetic circuits is inevitable for the long-term treatment of chronic UC. The problems associated with repeated injection should be dealt with properly. ${ }^{32}$ Therefore, we selected AAVs as the carrier of genetic circuits because AAVs are capable of establishing long-term transgene expression with minimal immunogenicity, toxicity and side effects. ${ }^{16} \mathrm{~A}$ growing number of human clinical trials have used AAVs, achieving a good safety profile and significant clinical benefit in many diseases. ${ }^{29}$ The results revealed that AAV-mediated liver uptake of the AAV-CMV-siR ${ }^{T+B+1}$ circuit caused substantial and lasting inhibition of the corresponding target genes in a chronic $U C$ model. The therapeutic benefit was comparable between the single injection of the AAV-CMV-siR ${ }^{T+B+1}$ circuit and repeated injection of the $C M V-s i R^{T+B+1}$ circuit. Therefore, even if more work is needed to verify the therapeutic effects and ensure safety, AAV-driven genetic circuits hold strong promise of becoming a new option for complex human diseases in the future.

Taken together, this study induced controllable and predictable self-assembly and delivery of multiple siRNAs in mouse models of UC and allowed simultaneous control of multiple immunological targets in a purpose-driven mode. This state-of-the-art technology has major theoretical significance and translational value because it provides a feasible and promising solution for combination therapy in UC.

\section{Declarations}

Disclosures: The authors have no potential conflicts to disclose.

Author contributions: Xi Chen, Chenyu Zhang, Xinyan Zhou and Zheng Fu conceived and designed the experiments. Xinyan Zhou, Mengchao Yu and Jinyu Fu performed the experiments. Yong Fu contributed the materials. Xinyan Zhou, Luzhen Ma, Jingwei Guo and Jieqiong Lei analyzed the data. Xi Chen and Chenyu Zhang contributed to the discussion. Xi Chen and Xinyan Zhou wrote the manuscript. 


\section{Abbreviations}

AAV, adeno-associated virus; AIDS, acquired immune deficiency syndrome; APCs, antigen presenting cells; bsAbs, bispecific antibodies; DAl, disease activity index; DSS, dextran sulphate sodium; FISH, fluorescence in situ hybridization; IBD, inflammatory bowel disease; ICAM, intercellular cellular adhesion molecules; IFN-y, interferon gamma; IFX, infliximab; LPMCs, lamina propria mononuclear cells; LPS, lipopolysaccharides; MAdCAM-1, mucosal addressin cellular adhesion molecule-1; MHC, major histocompatibility complex; MPO, myeloperoxidase; NTA, nanoparticle tracking analysis; PBLs, peripheral blood lymphocytes; PBMCs, peripheral blood mononuclear cells; RNAi, RNA interference; SLs, spleen lymphocytes; SMCs, spleen mononuclear cells; TEM, transmission electron microscopy; TNBS, trinitro-Benzenesulfonic acid; TNF-a, tumor necrosis factor alpha; UC, ulcerative colitis.

\section{References}

1. Ramos, G. P. \& Papadakis, K. A. Mechanisms of Disease: Inflammatory Bowel Diseases. Mayo Clinic Proceedings 94, 155-165, doi:10.1016/j.mayocp.2018.09.013 (2019).

2. Ungaro, R., Mehandru, S., Allen, P. B., Peyrin-Biroulet, L. \& Colombel, J.-F. Ulcerative colitis. Lancet 389, 1756-1770, doi:10.1016/s0140-6736(16)32126-2 (2017).

3. Neurath, M. F., Finotto, S. \& Glimcher, L. H. The role of Th1/Th2 polarization in mucosal immunity. Nat. Med. 8, 567-573, doi:10.1038/nm0602-567 (2002).

4. Rutgeerts, P. et al. Infliximab for induction and maintenance therapy for ulcerative colitis. New England Journal of Medicine 353, 2462-2476, doi:10.1056/NEJMoa050516 (2005).

5. Knight, D. M. et al. Construction and initial characterization of a mouse-human chimeric anti-TNF antibody. Molecular Immunology 30, 1443-1453, doi:10.1016/0161-5890(93)90106-I (1993).

6. Erle, D. J. et al. Expression and function of the MAdCAM-1 receptor, integrin alpha 4 beta 7, on human leukocytes. Journal of Immunology 153, 517-528 (1994).

7. Gordon, F. H., Pounder, R. E., Amlot, P. L. \& Donoghue, S. The effect of natalizumab, a humanized monoclonal antibody to alpha 4 integrin on circulating activated leucocytes in active inflammatory bowel disease (IBD). Gastroenterology 122, A434-A434 (2002).

8. Polese, L. et al. Role of CD40 and B7 costimulators in inflammatory bowel diseases. Acta bio-medica: Atenei Parmensis 74 Suppl 2, 65-70 (2003).

9. Grose, R. H., Howarth, G. S., Xian, C. J. \& Hohmann, A. W. Expression of B7 costimulatory molecules by cells infiltrating the colon in experimental colitis induced by oral dextran sulfate sodium in the mouse. Journal of Gastroenterology and Hepatology 16, 1228-1234, doi:10.1046/j.14401746.2001.02558.x (2001).

10. Schnitzler, F. et al. Long-term outcome of treatment with infliximab in 614 patients with Crohn's disease: results from a single-centre cohort. Gut 58, 492500, doi:10.1136/gut.2008.155812 (2009).

11. van Gennep, S. et al. High Disease Burden Drives Indirect Costs in Employed Inflammatory Bowel Disease Patients: The WORK-IBD Study. Inflammatory Bowel Diseases 27, 352-363, doi:10.1093/ibd/izaa082 (2021).

12. Kaplan, G. \& Panaccione, R. Use of Combination Therapy in IBD. Inflammatory Bowel Diseases 14, S219-S221, doi:10.1002/ibd.20688 (2008).

13. Kuehbacher, T., Abu Hashem, R., Langel, N., Schreiber, S. \& Drvarov, O. Combination therapy of vedolizumab and a TNF antagonist in IBD patients with severe chronic active, therapy refractory disease course. J. Crohns Colitis 11, S357-S357 (2017).

14. Mokhtari, R. B. et al. Combination therapy in combating cancer. Oncotarget 8, 38022-38043, doi:10.18632/oncotarget.16723 (2017).

15. Olesen, M. T. J., Ballarin-Gonzalez, B. \& Howard, K. A. The application of RNAi-based treatments for inflammatory bowel disease. Drug Delivery and Translational Research 4, 4-18, doi:10.1007/s13346-013-0156-9 (2014).

16. Liu, F. et al. Current Transport Systems and Clinical Applications for Small Interfering RNA (siRNA) Drugs. Mol. Diagn. Ther. 22, 551-569, doi:10.1007/s40291-018-0338-8 (2018).

17. Barile, L. \& Vassalli, G. Exosomes: Therapy delivery tools and biomarkers of diseases. Pharmacology \& Therapeutics 174, 63-78, doi:10.1016/j.pharmthera.2017.02.020 (2017).

18. Fu, Z. et al. In vivo self-assembled small RNAs as a new generation of RNAi therapeutics. Cell Research, doi:10.1038/s41422-021-00491-z (2021).

19. Ray, A. \& Dittel, B. N. Isolation of mouse peritoneal cavity cells. Journal of visualized experiments: JoVE, doi:10.3791/1488 (2010).

20. Rogler, G. et al. Isolation and phenotypic characterization of colonic macrophages. Clinical and Experimental Immunology 112, 205-215 (1998).

21. Fais, S. et al. Spontaneous release of interferon gamma by intestinal lamina propria lymphocytes in Crohn's disease. Kinetics of in vitro response to interferon gamma inducers. Gut 32, 403-407, doi:10.1136/gut.32.4.403 (1991).

22. Hueber, W. et al. Secukinumab, a human anti-IL-17A monoclonal antibody, for moderate to severe Crohn's disease: unexpected results of a randomised, double-blind placebo-controlled trial. Gut 61, 1693-1700, doi:10.1136/gutjnl-2011-301668 (2012).

23. Nava, P. et al. Interferon-gamma Regulates Intestinal Epithelial Homeostasis through Converging beta-Catenin Signaling Pathways. Immunity 32, 392402, doi:10.1016/j.immuni.2010.03.001 (2010).

24. Sarra, M. et al. Interferon-gamma-expressing Cells Are a Major Source of Interleukin-21 in Inflammatory Bowel Diseases. Inflammatory Bowel Diseases 16, 1332-1339, doi:10.1002/ibd.21238 (2010).

25. Gerlach, K. et al. Transcription Factor NFATc2 Controls the Emergence of Colon Cancer Associated with IL-6-Dependent Colitis. Cancer Research 72, 43404350, doi:10.1158/0008-5472.Can-11-4155 (2012). 
26. Zundler, S. et al. Blockade of alpha E beta 7 integrin suppresses accumulation of CD8(+) and Th9 lymphocytes from patients with IBD in the inflamed gut in vivo. Gut 66, 1936-1948, doi:10.1136/gutjnl-2016-312439 (2017).

27. Rogler, G. et al. T-cell co-stimulatory molecules are upregulated on intestinal macrophages from inflammatory bowel disease mucosa. European Journal of Gastroenterology \& Hepatology 11, 1105-1111, doi:10.1097/00042737-199910000-00006 (1999).

28. Thornton, A. M. \& Shevach, E. M. CD4(+)CD25(+) immunoregulatory T cells suppress polyclonal T cell activation in vitro by inhibiting interleukin 2 production. Journal of Experimental Medicine 188, 287-296, doi:10.1084/jem.188.2.287 (1998).

29. Wang, D., Tai, P. W. L. \& Gao, G. Adeno-associated virus vector as a platform for gene therapy delivery. Nature Reviews Drug Discovery $18,358-378$, doi:10.1038/s41573-019-0012-9 (2019).

30. Baker, H. Combination therapy for IBD. Lancet Gastroenterology \& Hepatology 5, 340-340 (2020).

31. Panaccione, R. et al. Combination Therapy With Infliximab and Azathioprine Is Superior to Monotherapy With Either Agent in Ulcerative Colitis. Gastroenterology 146, 392-+, doi:10.1053/j.gastro.2013.10.052 (2014).

32. Sakurai, H., Kawabata, K., Sakurai, F., Nakagawa, S. \& Mizuguchi, H. Innate immune response induced by gene delivery vectors. International Journal of Pharmaceutics 354, 9-15, doi:10.1016/j.jpharm.2007.06.012 (2008).

\section{Figures}

a
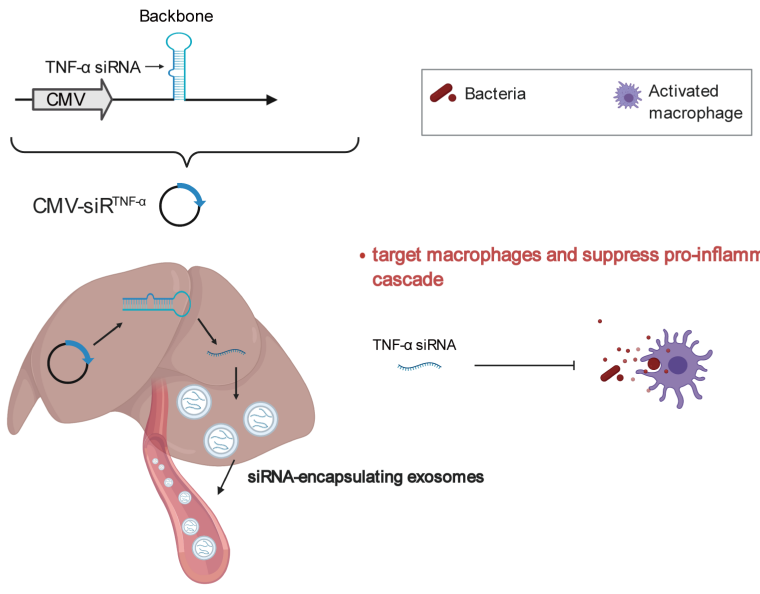

b
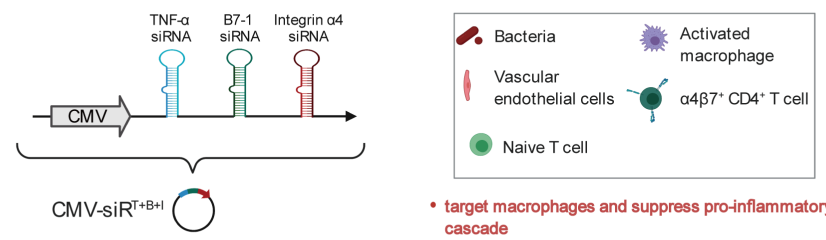

- target macrophages and suppress pro-inflammatory cascad

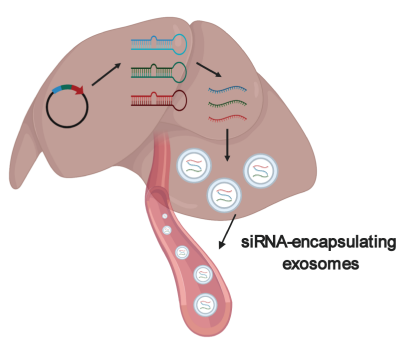

TNF-a siRNA

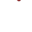

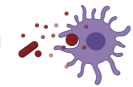

- target antigen presenting cells (e.g. macrophage) and inhibit co-stimulatory signal to T cells

B7-1 siRNA

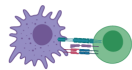

- target $\mathrm{\alpha} 4 \beta 7^{+} \mathrm{CD} 4^{+} \mathrm{T}$ cells and block $\mathrm{T}$ cell homing

to inflamed sites

Integrin a4 siRNA

egrin a4 siRNA

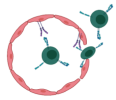

\section{Figure 1}

Schematic description of the architecture of the genetic circuits. (a) The CMV-siRTNF-a circuit contains a CMV promoter part and a TNF-a siRNA-expressing part. When the CMV-siRTNF-a circuit is taken up and processed by the liver after intravenous injection, the CMV promoter drives the transcription of TNF-a siRNA and directs the loading of TNF-a siRNA into exosomes as cargo. Subsequently, TNF-a siRNA-encapsulating exosomes facilitate the systematic distribution of siRNAs to multiple tissues and cells, including colonic macrophages. Once arriving colonic macrophages, TNF- $a$ siRNA has the potential to regulate immune balance and relieve intestinal inflammation by inhibiting the uncontrolled release of TNF-a from macrophages. (b) The multitargeted CMVSiRT+B+l circuit carries three siRNA expression cassettes, which are organized into a head-to-tail tandem array under the control of a CMV promoter, to simultaneously express TNF-a siRNA, B7-1 siRNA and integrin a4 siRNA. When these three siRNAs are loaded into exosomes and delivered to appropriate 
immune cells, TNF-a siRNA can suppress the proinflammatory cascade, B7-1 siRNA can inhibit the costimulatory signal to T cells, and integrin a4 siRNA can block T cell homing to the sites of inflammation, thereby exerting a synergistic therapeutic effect against UC.
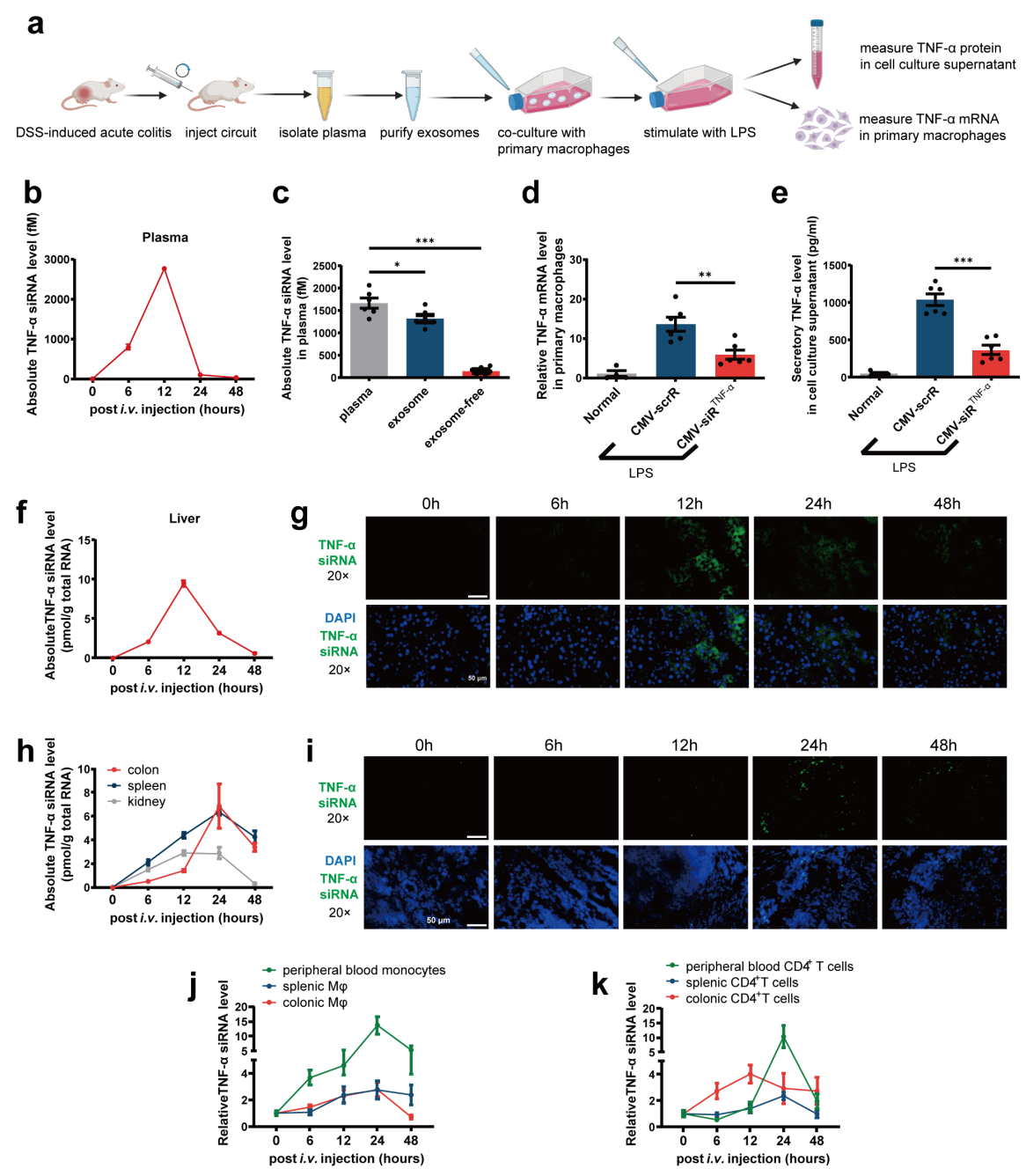

Figure 2

Characterization of the self-assembly of TNF-a siRNA-encapsulating exosomes in an ex vivo model. (a) Schematic of the experimental design. Acute UC was induced in male BALB/c mice by replacing their drinking water with a $2.5 \%$ DSS solution for 7 days. DSS mice were intravenously injected with 5 mg/kg CMVscrR or CMV-siRTNF-a every day for a total of 7 times, and the exosomes were then purified from mouse plasma and incubated with primary macrophages. After stimulating macrophages with $50 \mathrm{ng} / \mathrm{ml}$ LPS, the uptake of TNF-a siRNA-encapsulating exosomes by macrophages and subsequent suppression of TNF-a expression by TNF-a siRNA were examined in this ex vivo model. (b) Kinetics of TNF-a siRNA in mouse plasma following tail vein injection of the 5 $\mathrm{mg} / \mathrm{kg}$ CMV-siRTNF-a circuit ( $\mathrm{n}=3$ in each group). (c) Quantitative RT-PCR analysis of the absolute expression levels of TNF-a siRNA in plasma, exosome and exosome-free plasma ( $n=6$ in each group). (d) Quantitative RT-PCR analysis of the relative expression levels of TNF-a mRNA in primary macrophages ( $n=6$ in each group). (e) Determination of the levels of secretory TNF-a protein in cell culture supernatant by ELISA ( $n=6$ in each group). (f) Kinetics of TNF-a siRNA in mouse liver following tail vein injection of the $5 \mathrm{mg} / \mathrm{kg}$ CMV-siRTNF-a circuit ( $\mathrm{n}=3$ in each group). (g) In situ detection of TNF-a siRNA in liver sections of DSS mice at $0,6,12,24$ or 48 hours after injection with the CMV-siRTNF-a circuit. Positive in situ hybridization signals are shown in green, and DAPI-stained nuclei are shown in blue. Scale bar: $50 \mu \mathrm{m}$. (h) Kinetics of TNF-a siRNA in the mouse colon, spleen and kidney following tail vein injection of the $5 \mathrm{mg} / \mathrm{kg}$ CMV-siRTNF-a circuit ( $\mathrm{n}=3$ in each group). (i) In situ detection of TNF-a siRNA in colon sections of DSS mice at $0,6,12,24$ or 48 hours after injection with the CMV-siRTNF-a circuit. Positive in situ hybridization signals are shown in green, and DAPI-stained nuclei are shown in blue. Scale bar: $50 \mu \mathrm{m}$. (j) Kinetics of TNF-a siRNA in monocytes and macrophages derived from peripheral blood, spleen and colonic lamina propria of DSS mice following tail vein injection of the $5 \mathrm{mg} / \mathrm{kg}$ CMV-siRTNF-a circuit ( $\mathrm{n}=3$ in each group). ( $\mathrm{k}$ ) Kinetics of TNF-a siRNA in CD4+ T cells derived from peripheral blood, spleen and colonic lamina propria of DSS mice following tail vein injection of the $5 \mathrm{mg} / \mathrm{kg}$ CMV-siRTNF-a circuit ( $\mathrm{n}=3$ in each group). Values are presented as the mean \pm SEM. Significance was determined using one-way ANOVA followed by Dunnett's multiple comparison in panels $c, d$ and $e .{ }^{*} p<0.05 ; * \star p<0.01 ; * \star \star p<0.005$. 
a

construct acute UC model

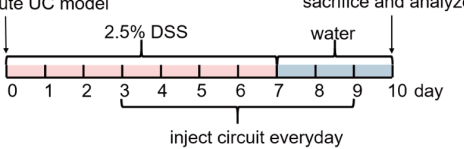

b

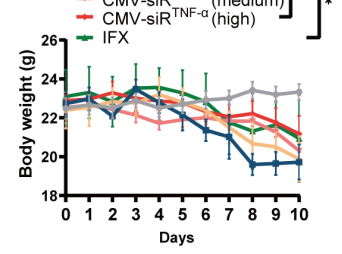

- Normal

- CMV-scrR
- CMV-siR
TNF-a $($ low)

- CMV-siR TNF-a (me

Days

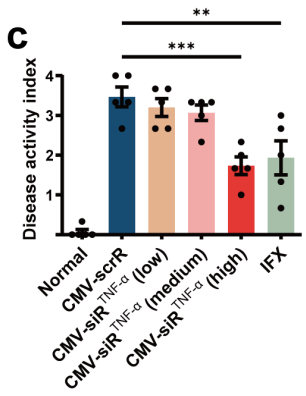

d

e
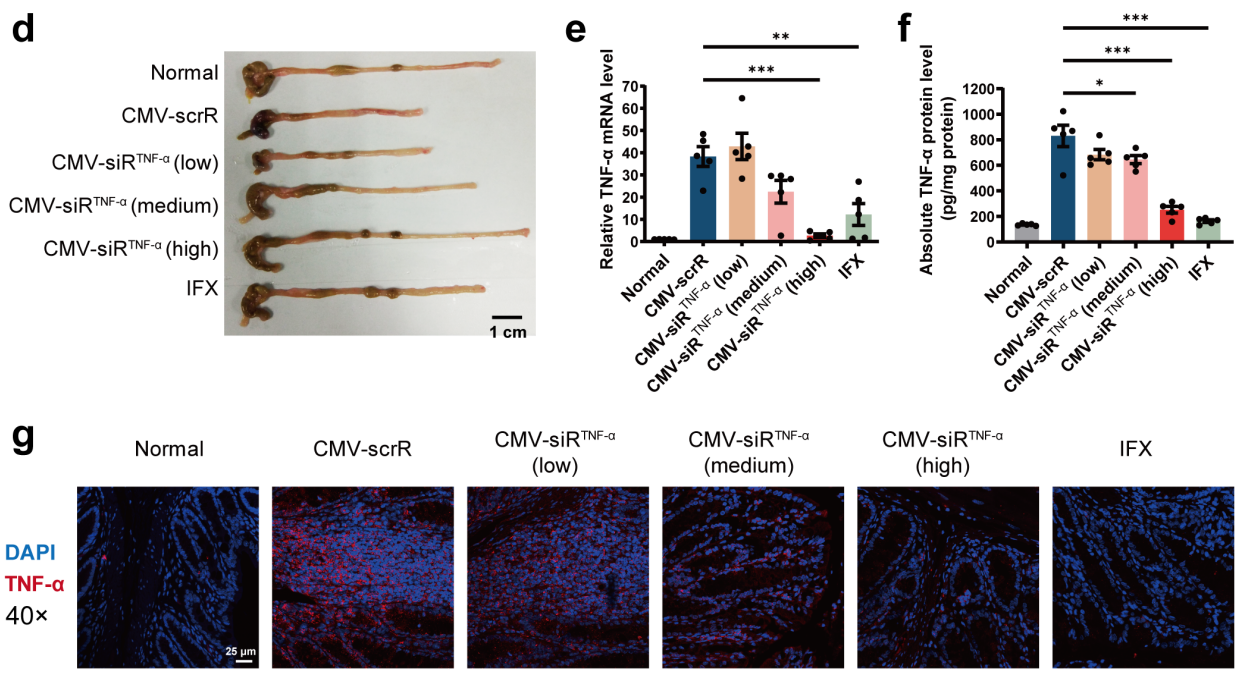

CMV-siR ${ }^{\mathrm{TNF}-\mathrm{a}}$

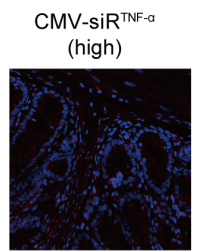

IFX

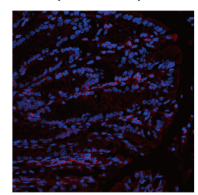

CMV-SiR ${ }^{\text {TNF-a }}$ (low)

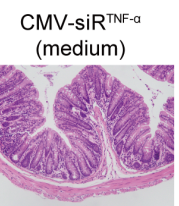

CMV-siR ${ }^{\text {TNF- } \alpha}$ (high)

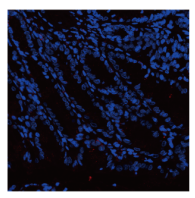

h

CMV-scrR

$20 x$
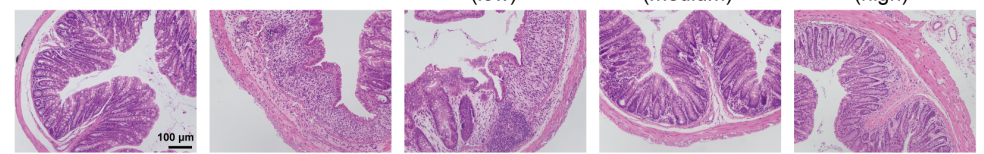

IFX

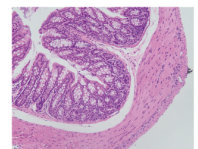

Figure 3

Intravenous injection of the CMV-siRTNF-a circuit protects mice from DSS-induced acute UC. (a) Flow chart of the experimental design. Acute UC was induced in male BALB/c mice by replacing their drinking water with a $2.5 \%$ DSS solution for 7 days. From day 3 of modelling, the mice were intravenously injected with $20 \mathrm{mg} / \mathrm{kg} \mathrm{CMV}$-scrR or three dosages $(0.5,5$ and $20 \mathrm{mg} / \mathrm{kg}$ ) of the CMV-siRTNF-a circuit or $20 \mathrm{mg} / \mathrm{kg}$ infliximab (IFX) once a day. After 7 injections, the mice were euthanized. Body weights were monitored daily, and symptoms and histology were evaluated on day 10 . Untreated BALB/c mice were included as normal controls. (b) Body weight curves ( $n=5$ in each group). (c) DAl scores ( $n=5$ in each group). (d) Representative macroscopic features of colons. Scale bar: 1 $\mathrm{cm}$. (e) Quantitative RT-PCR analysis of the relative expression levels of TNF-a mRNA in the colon ( $\mathrm{n}=5$ in each group). (f) Determination of the absolute expression levels of TNF-a protein in the colon by ELISA ( $n=5$ in each group). (g) Immunofluorescence staining of TNF-a (red) and DAPI (blue) in colon sections. Scale bar: $25 \mu \mathrm{m}$. (h) Representative images of H\&E staining of colon sections. Scale bar: $100 \mu \mathrm{m}$. Values are presented as the mean \pm SEM. Significance was determined using one-way ANOVA followed by Dunnett's multiple comparison in panels c, e and f or two-way ANOVA followed by Dunnett's multiple comparison in panel b. ${ }^{*} p<0.05 ;{ }^{* \star} p<0.01$; ${ }^{* \star} p<0.005$. 
a
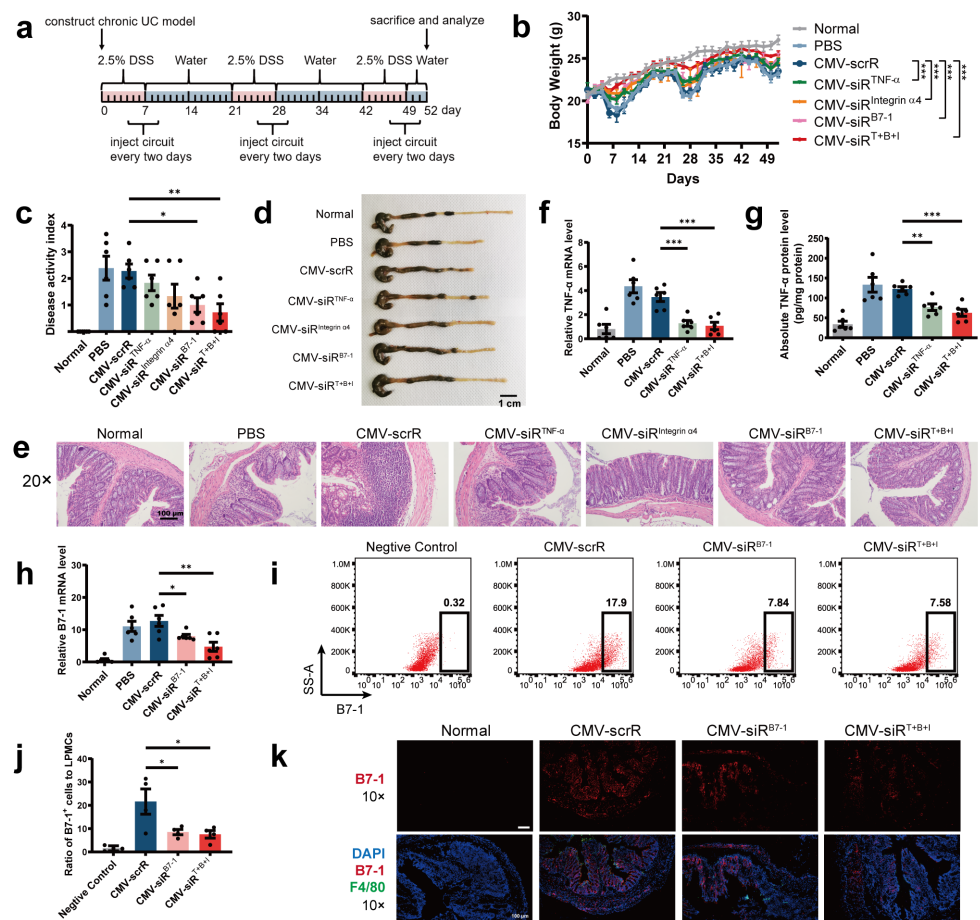

k
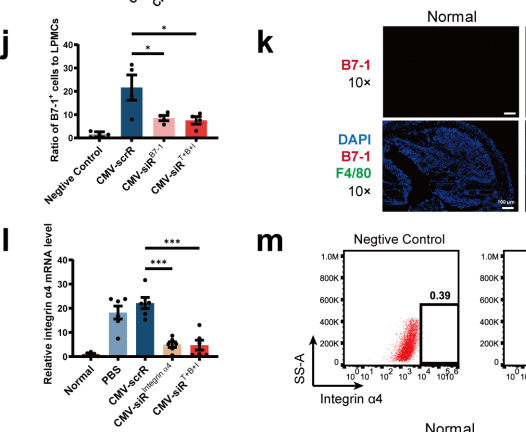

n
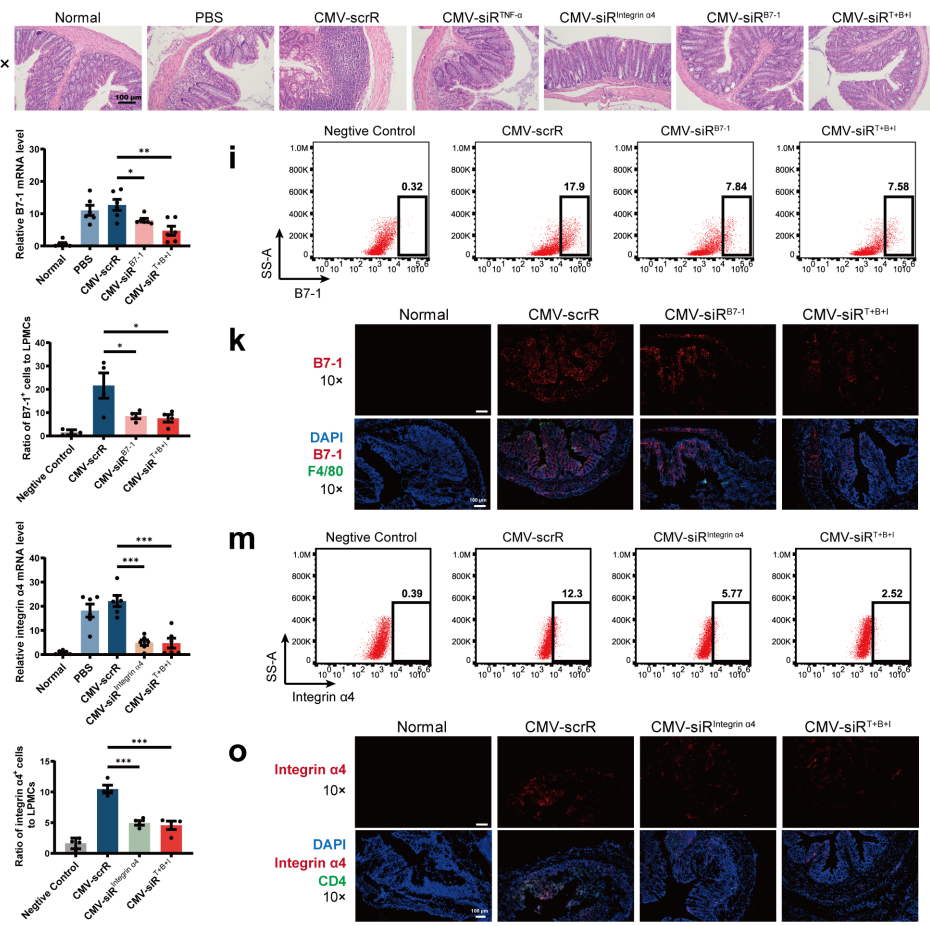

CMV-siptost

0
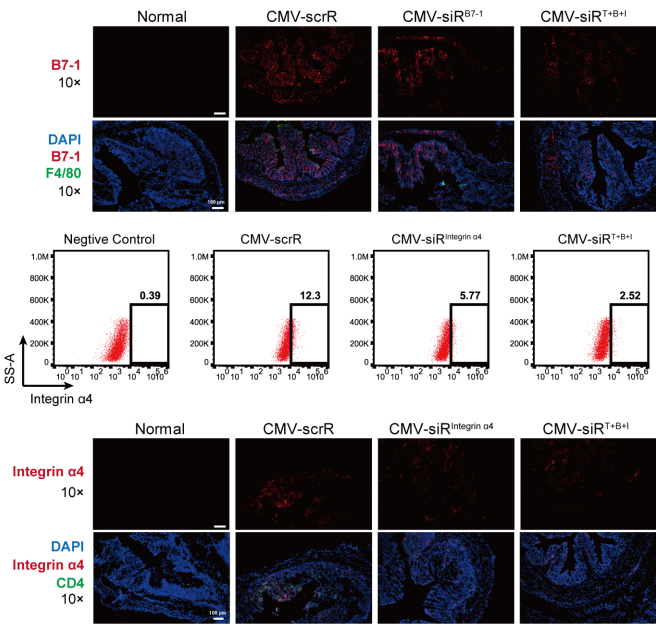

CMV-siR Tr+s+1 $^{2}$
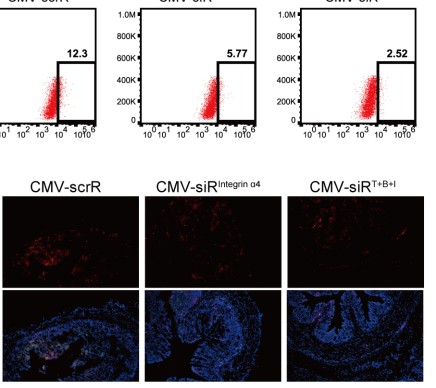

Figure 4

Intravenous injection of the multitargeted CMV-siRT+B+I circuit simultaneously inhibits TNF-a, B7-1 and integrin a4 in target cells and exerts a synergistic therapeutic effect against DSS-induced chronic UC. (a) Flow chart of the experimental design. Chronic UC was induced in male BALB/c mice by rhythmically administering to mice $2.5 \%$ DSS for 1 week and water for 2 weeks for 3 cycles. Four days after each DSS drinking, mice were intravenously injected with PBS, $10 \mathrm{mg} / \mathrm{kg}$ CMV-scrR, $10 \mathrm{mg} / \mathrm{kg}$ CMV-siRTNF-a, $10 \mathrm{mg} / \mathrm{kg}$ CMV-siRIntegrin a4, $10 \mathrm{mg} / \mathrm{kg}$ CMV-siRB7-1 or $10 \mathrm{mg} / \mathrm{kg}$ CMV-siRT+B+l circuit for a total of 3 times, once every 2 days. Two days after the final injection on day 52, the mice were euthanized. Body weights were monitored every two days, and symptoms and histology were evaluated on day 52. Untreated BALB/c mice were included as normal controls. (b) Body weight curves ( $n=6$ in each group). (c) DAl scores ( $n$ $=6$ in each group). (d) Representative macroscopic features of colons. Scale bar: $1 \mathrm{~cm}$. (e) Representative images of H\&E staining of colon sections. Scale bar: $100 \mu \mathrm{m}$. (f) Quantitative RT-PCR analysis of the relative expression levels of TNF-a mRNA in the colon ( $\mathrm{n}=6$ in each group). ( $\mathrm{g}$ ) Determination of the absolute expression levels of TNF-a protein in the colon by ELISA ( $n=6$ in each group). (h) Quantitative RT-PCR analysis of the relative expression levels of B71 mRNA in the colon ( $n=6$ in each group). (i) Representative flow cytometric plots of B7-1 on the surface of colonic lamina propria mononuclear cells. (j) The population of B7-1+ cells in total colonic lamina propria mononuclear cells ( $n=4$ in each group). (k) Immunofluorescence staining of B7-1 (red), F4/80 (green) and DAPI (blue) in colon sections. Double-positive (red and green) signals indicate B7-1+ macrophages. Scale bar: $100 \mu \mathrm{m}$. (I) Quantitative RT-PCR analysis of the relative expression levels of integrin a4 mRNA in the colon ( $n=6$ in each group). (m) Representative flow cytometric plots of integrin a4 on the surface of mononuclear cells derived from the colonic lamina propria. $(n)$ The population of integrin a4+ lymphocytes in total colonic lamina propria lymphocytes $(n=4$ in each group). (o) Immunofluorescence staining of integrin a4 (red), CD4 (green) and DAPI (blue) in colon sections. Double-positive (red and green) signals indicate integrin a4+CD4+ cells. Scale bar: $100 \mu \mathrm{m}$. Values are presented as the mean \pm SEM. Significance was determined using one-way ANOVA followed by Dunnett's multiple comparison in panels $\mathrm{c}, \mathrm{f}, \mathrm{g}, \mathrm{h}, \mathrm{j}, \mathrm{I}$ and $\mathrm{n}$ or two-way ANOVA followed by Dunnett's multiple comparison in panel $\mathrm{b} .{ }^{*} \mathrm{p}<0.05$; $* \star \mathrm{p}<0.01$; $* * * p 0.005$. 

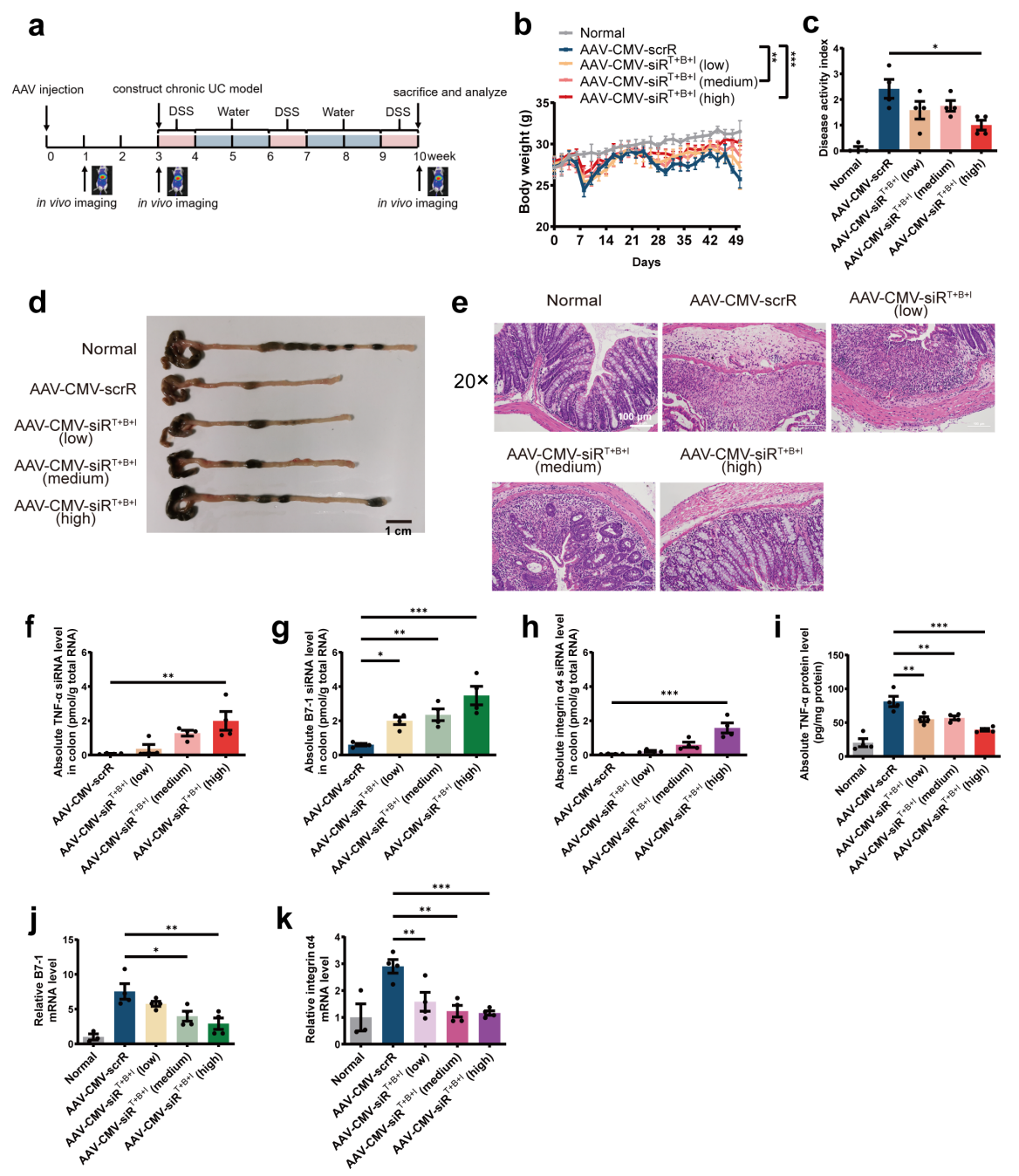

Figure 5

Intravenous injection of the AAV-CMV-siRT+B+I circuit induces long-term combination therapy in the DSS-induced chronic UC model. (a) Flow chart of the experimental design. On week 0, BALB/c mice were intravenously injected with AAV-CMV-scrR (1.0×1011 v.g.) or with three dosages of AAV-CMV-siRT+B+I $(2.5 \times 1010$ v.g., $5.0 \times 1010$ v.g. or $1.0 \times 1011$ v.g.). Three weeks later, chronic UC was induced by rhythmically administering to mice $2.5 \%$ DSS for 1 week and water for 2 weeks. Body weights were monitored every two days, and the symptoms and histology were evaluated at week 10 . Untreated BALB/c mice were included as normal controls. (b) Body weight curves ( $n=4$ in each group). (c) DAl scores ( $n=4$ in each group). (d) Representative macroscopic features of colons. Scale bar: $1 \mathrm{~cm}$. (e) Representative images of H\&E staining of colon sections. Scale bar: $100 \mu \mathrm{m}$. (f-h) Quantitative RT-PCR analysis of the absolute expression levels of TNF-a siRNA ( $f$ ), B7-1 siRNA (g) and integrin a4 siRNA ( $h$ ) in the colon ( $n=4$ in each group). (i) Determination of the absolute expression levels of TNF-a protein in the colon by ELISA ( $n=4$ in each group). ( $j$ and $k$ ) Quantitative RT-PCR analysis of the relative expression levels of B7-1 mRNA (j) and integrin a4 mRNA $(k)$ in the colon ( $n=4$ in each group). Values are presented as the mean \pm SEM. Significance was determined using one-way ANOVA followed by Dunnett's multiple comparison in panels $\mathrm{c}, \mathrm{f}, \mathrm{g}, \mathrm{h}, \mathrm{i}, \mathrm{j}$, and k or two-way ANOVA followed by Dunnett's multiple comparison in panel b. * $\mathrm{p}<0.05$; ** $p<0.01 ; * * * p<0.005$.

\section{Supplementary Files}

This is a list of supplementary files associated with this preprint. Click to download.

- supplementaryfile.docx 